\title{
On minima of a functional of the gradient: Upper and lower solutions
}

\author{
Vladimir V. Goncharov*,1, António Ornelas \\ CIMA-UE, rua Romão Ramalho 59, P-7000-671 Évora, Portugal
}

Received 2 April 2005; accepted 1 July 2005

\begin{abstract}
This paper studies a scalar minimization problem with an integral functional of the gradient under affine boundary conditions. A new approach is proposed using a minimal and a maximal solution to the convexified problem. We prove a density result: any relaxed solution continuously depending on boundary data may be approximated uniformly by solutions of the nonconvex problem keeping continuity relative to data. We also consider solutions to the nonconvex problem having Lipschitz dependence on boundary data with the best Lipschitz constant.
\end{abstract}

(c) 2005 Elsevier Ltd. All rights reserved.

Keywords: Scalar variational problem; Nonconvex lagrangian; Baire category theorem; Continuous selection; Relaxation

\section{Introduction}

Consider the integral functional

$$
J(u)=\int_{\Omega} g(\nabla u(x)) \mathrm{d} x,
$$

\footnotetext{
is The research leading to this paper was financially supported by: projecto POCTI/1999/Mat/36422 and Financiamento Programático Especial do CIMA-UE (Centro de Investigação em Matemática e Aplicações da Universidade de Évora, Portugal) both of FCT (Fundação para a Ciência e Tecnologia, Portugal).

* Corresponding author. Tel.: +351936 514934; fax: +351 266744971.

E-mail addresses: goncha@uevora.pt (V.V. Goncharov), ornelas@uevora.pt (A. Ornelas).

${ }^{1}$ V.V.G. also belongs to the Irkutsk Institute of Systems Dynamics and Control Theory, Siberian Department of RAS, ul. Lermontov 134, 664033 Irkutsk, Russia.
} 
defined on the class of scalar Sobolev functions subject to a boundary condition $\left.u\right|_{\partial \Omega}=u_{0}(\cdot)$. Here $\Omega$ is an open bounded set in $\mathbb{R}^{n}$ and the lagrangian $g: \mathbb{R}^{n} \rightarrow \mathbb{R} \cup\{+\infty\}$ is lower semicontinuous, possibly nonconvex. In applications to elasticity theory, the functional $J$ can be the free energy of a homogeneous body undergoing antiplane shear deformations.

In the past many researchers have paid attention to the problem of minimizing such integral, under various physically reasonable hypotheses on the lagrangian and on boundary data. Necessary and sufficient conditions for the existence of minimizers, in the case of affine data, were independently found by Cellina and by Friesecke in the beginning of the nineties (see $[3,4,14])$. They have shown that existence or nonexistence of minimizers for the problem

$$
\min \left\{\int_{\Omega} g(\nabla u(x)) \mathrm{d} x: u(\cdot) \in\langle v, \cdot\rangle+\mathbf{W}_{0}^{1, p}(\Omega)\right\}
$$

depends on the position of the vector $v$ on the geometrical structure of the lagrangian. More precisely, denoting by $g^{* *}(\cdot)$ the bipolar function, i.e. the greatest convex lower semicontinuous function below $g(\cdot)$, the problem $\left(\mathscr{P}_{v}\right)$ admits at least one solution if and only if

$$
\begin{aligned}
& \text { either } g^{* *}(v)=g(v) \\
& \text { or } \\
& \text { near the point } v \text { the function } g^{* *}(\cdot) \text { is affine. }
\end{aligned}
$$

Notice that in [3,4] the author supposed superlinear growth of $g$, while in [14] the lagrangian was assumed continuous. In case $g$ admits values $+\infty$ in the condition above one must assume $v$ to be an interior point of the effective domain of $g^{* *}$.

Later (see $[20,21]$ ) the same variational problem with a more general boundary condition was considered

$$
\min \left\{\int_{\Omega} g(\nabla u(x)) \mathrm{d} x: u(\cdot) \in u_{0}(\cdot)+\mathbf{W}_{0}^{1, p}(\Omega)\right\}
$$

and a condition for its solvability, for each function $u_{0}(\cdot)$ having enough regularity, was proposed. The technique was mainly inspired by research on boundary value problems for Hamilton-Jacobi equations (see [12]). It also contains an original argument using the differentiability (a.e.) of a solution to the relaxed problem. In order to have this property one needs to assume that either $p>n$ or the lagrangian satisfies a rather strong growth hypothesis (see [20]).

Let us return now to the problem $\left(\mathscr{P}_{v}\right)$. The next natural question is stability of solutions w.r.t. boundary data. Such kind of result was obtained, e.g., in [5]. Fixing a $v$ for which there exists a unique solution $u_{v}(\cdot)$, the authors studied convergence of various sequences of solutions $u_{v_{n}}(\cdot)$ associated to vectors $v_{n} \rightarrow v$ as $n \rightarrow \infty$. Since, in general, we have no uniqueness, then other question is to find a minimizer depending on $v$ in a suitable (continuous) way. This question was first considered in [15], where the authors proved the 
existence of a continuous mapping $s(v)(x)$ such that: $s(v)(\cdot)$ solves $\left(\mathscr{P}_{v}\right)$ for almost any $v$ satisfying the condition $(\mathbb{C})$ while for other $v$ it always minimizes the relaxed functional. A stronger result was reached later, in [10], constructing a Lipschitz continuous selection associating to each $v$ a minimizer of the original nonconvex problem $\left(\mathscr{P}_{v}\right)$, at those $v$ where it exists. Notice that on the set of minimizers we have the uniform topology induced by the space $\mathbf{C}(\bar{\Omega})$.

The common technical tool which unifies all of the above results is the Vitali covering theorem. Roughly speaking, this approach permits us to obtain a solution of the original problem by a series of local perturbations of a solution of the convexified one. Its application is much simpler in the case of affine boundary data, due to the fact that the function $x \mapsto\langle v, x\rangle$ itself is a solution of the relaxed problem. Thus, given a suitable $v$, a minimizer of $\left(\mathscr{P}_{v}\right)$ can be obtained as an arbitrarily small perturbation of the affine function. However, a simple application of the Vitali theorem is not enough if one wishes to keep the continuity of a solution w.r.t. $v$. That is why the paper [10] developed a constructive version of the Vitali method, taking into account also the dependence on $v$. However, this technique is not convenient to describe the family of all the solutions.

In this paper we develop a different approach, based on two functions which enclose the solutions of the relaxed problem. Assuming that there exist no unbounded $n$-dimensional faces (and this may be obtained by imposing e.g. superlinear growth), these functions (defined explicitly) are themselves solutions of the relaxed problem. Moreover, they are (Lipschitz) continuous as mappings from $\operatorname{dom}\left(g^{* *}\right)$ to $\mathbf{C}(\bar{\Omega})$. Then a simple compactness argument permits us to construct minimizers of the original nonconvex functional which approximate the upper and the lower solutions, keeping continuous dependence on $v$. Furthermore, in such a way one can approximate any minimizer $\tilde{s}(v)(\cdot)$ of the relaxed functional which satisfies either $\tilde{s}(v)(x)>\langle v, x\rangle$, or $\tilde{s}(v)(x)<\langle v, x\rangle$ for all $v$ and $x$ while for a more general minimizer (containing possibly affine pieces as well) we apply first some polynomial approximation. However, if the epigraph of the function $g^{* *}$ has unbounded $n$-dimensional faces, then the closure of the solution set of the original problem $\left(\mathscr{P}_{v}\right)$ is, in general, strictly included in the family of solutions of the relaxed one (as shown in Example 5.1).

The paper is organized as follows. In Section 2 after some preliminaries, we constructively define upper and lower functions, associated with the extremal structure of the epigraph of $g^{* *}$, and discuss their connection with the family of solutions of the convexified variational problem. Then (Section 3) we formulate the main theorem (on density of the solution set uniformly w.r.t. $v$ ) and introduce the basic constructions needed to prove it. As convenient tools to treat such kind of problems we use the Baire category theorem and a Choquet function characterizing extreme points of a convex compact set. In the following section we prove some auxiliary statements on polynomial approximations, while the essential part of the proof of the main theorem is contained in Section 5. In Section 6 we modify the proof in order to obtain the result on density of solutions which are Lipschitz continuous w.r.t. $v$. Finally, the last section analyses our variational problem in terms of a differential inclusion for the gradient, and shows a concrete example in which the lower and the upper solutions do not solve the nonconvex problem. 


\section{Preliminaries. Solutions of the relaxed problem}

In what follows we assume $\Omega$ to be an open bounded set in $\mathbb{R}^{n}$, and $g: \mathbb{R}^{n} \rightarrow \mathbb{R} \cup\{+\infty\}$ to be a lower semicontinuous proper function. The following notations will be used:

- $\operatorname{dom}(g):=\left\{v \in \mathbb{R}^{n}: g(v)<+\infty\right\}$ - the domain of $g(\cdot)$

- epi $(g):=\left\{(v, r) \in \mathbb{R}^{n} \times \mathbb{R}: r \geqslant g(v)\right\}$ - the epigraph of $g(\cdot)$

- $\operatorname{diam} A:=\sup \{|x-y|: x, y \in A\}$-the diameter of a set $A \subset \mathbb{R}^{n}$

- $\overline{\mathrm{co}} A$ - the convex closed hull of $A$

- ext $A$-the set of all extreme points of $A$

- int $A$-the interior of $A$

- $\partial A$ - the boundary of $A$

- $\bar{A}$ - the closure of $A$

- $\overline{\mathbf{B}}$ - the closed unit ball centered at zero in $\mathbb{R}^{n}$

- $\sigma_{A}(v):=\sup \{\langle v, x\rangle: x \in A\}$-the support function of a set $A \subset \mathbb{R}^{n}$ (where $\langle\cdot, \cdot\rangle$ is the scalar product in $\left.\mathbb{R}^{n}\right)$

- $A^{*}:=\left\{v \in \mathbb{R}^{n}: \sigma_{A}(v) \leqslant 1\right\}$ - the polar of a set $A \subset \mathbb{R}^{n}$

- $\operatorname{dist}_{A}(\cdot)$ - the distance from a point to a set $A \subset \mathbb{R}^{n}$

- $\mu(\Omega)$ - the $n$-dimensional Lebesgue measure of $\Omega$

- $\mathbf{C}(\bar{\Omega})$ - the space of all continuous functions $u: \bar{\Omega} \rightarrow \mathbb{R}$ supplied with the norm $\|u\|_{\infty}:=\sup _{x \in \bar{\Omega}}|u(x)|$

- $\mathbf{C}_{0}(\bar{\Omega})$ - the subspace of those $u(\cdot) \in \mathbf{C}(\bar{\Omega})$ which are zero on $\partial \Omega$.

We consider the bipolar function $g^{* *}: \mathbb{R}^{n} \rightarrow \mathbb{R} \cup\{+\infty\}$ whose epigraph is the closed convex hull of epi $(g)$. Since any convex set can be represented as the union of the relative interiors of its faces (see [17, p. 164]), to each vector $v \in \operatorname{dom}\left(g^{* *}\right)$ we can associate a unique (nonvertical) face $F(v)$ of epi $\left(g^{* *}\right)$ to whose relative interior the point $\left(v, g^{* *}(v)\right)$ belongs. Let us denote by $\hat{F}(v)$ the projection of this face into $\mathbb{R}^{n}$. One way to guarantee boundedness of all the sets $\hat{F}(v)$ is the following superlinear growth assumption:

$$
\lim _{|v| \rightarrow+\infty} \frac{g(v)}{|v|}=+\infty
$$

Another way is the hypothesis of strict convexity at infinity: there exist no nonvertical rays (i.e. straight half-lines) contained in the epigraph of $g^{* *}$. It may also be obtained by imposing a non-coercive growth condition: $g^{* *}\left(\xi_{k}\right)-\left\langle m_{k}, \xi_{k}\right\rangle \rightarrow-\infty$ for any sequence $\left\{\xi_{k}\right\} \subset \operatorname{dom}\left(g^{* *}\right)$ having $\left|\xi_{k}\right| \rightarrow \infty$ and $m_{k} \in \partial g^{* *}\left(\xi_{k}\right) \quad \forall k$.

But even when these hypotheses are violated, one can write the necessary and sufficient condition of existence of a minimizer in $\left(\mathscr{P}_{v}\right)$, for $v \in \operatorname{int} \operatorname{dom}\left(g^{* *}\right)$, as

$$
v \in Y \cup \operatorname{int} \overline{\operatorname{co}}(Y \cap \hat{F}(v))
$$

where $Y:=\left\{v \in\right.$ int $\left.\operatorname{dom}\left(g^{* *}\right): g(v)=g^{* *}(v)\right\}$. In [20] this condition was formulated in terms of the subdifferential of $g$ (see Theorem 1.1 there). 
Let us consider now the relaxed (convexified) variational problem

$$
\min \left\{\int_{\Omega} g^{* *}(\nabla u(x)) \mathrm{d} x: u(\cdot) \in\langle v, \cdot\rangle+\mathbf{W}_{0}^{1, p}(\Omega)\right\} .
$$

This problem always admits a solution (for instance, the affine function $\langle v, x\rangle$ itself), and the minimum is $g^{* *}(v) \mu(\Omega)$. By the relaxation result [20, Lemma 2.3] we have also

$$
\inf \left\{\int_{\Omega} g(\nabla u(x)) \mathrm{d} x: u(\cdot) \in\langle v, \cdot\rangle+\mathbf{W}_{0}^{1, p}(\Omega)\right\}=g^{* *}(v) \mu(\Omega),
$$

whenever $v \in \operatorname{int} \operatorname{dom}\left(g^{* *}\right)$. Recall that for any solution $u(\cdot)$ of $\left(\mathscr{R} \mathscr{P}_{v}\right)$ the inclusion

$$
\nabla u(x) \in \hat{F}(v)
$$

holds for almost every (a.e.) $x \in \Omega$. Moreover, by the divergence theorem (see, e.g., [13, p. 478]) it follows that each function $u(\cdot) \in\langle v, \cdot\rangle+\mathbf{W}_{0}^{1, p}(\Omega)$ satisfying the condition (2.1) is indeed a minimizer of the problem $\left(\mathscr{R} \mathscr{P}_{v}\right)$. Notice that this property does not depend neither on the smoothness of the boundary $\partial \Omega$ nor on the growth of the integrand (see the proof of Lemma 3.3 in [14]). However, for the sake of simplicity, further on we suppose the hypothesis $(\mathbb{M})$ or strict convexity at infinity to be satisfied. The condition $\left(\mathbb{C}^{\prime}\right)$ in this case, by the Krein-Milman theorem, takes the form

$$
v \in Y \cup \operatorname{int} \hat{F}(v) \text {. }
$$

Let us start by defining the functions as following:

$$
\begin{aligned}
& s^{+}(v)(x):=\inf _{x^{\prime} \in \partial \Omega} \sup _{v^{\prime} \in \hat{F}(v)}\left\{\left\langle v, x^{\prime}\right\rangle+\left\langle v^{\prime}, x-x^{\prime}\right\rangle\right\}, \\
& s^{-}(v)(x):=\sup _{x^{\prime} \in \partial \Omega} \inf _{v^{\prime} \in \hat{F}(v)}\left\{\left\langle v, x^{\prime}\right\rangle+\left\langle v^{\prime}, x-x^{\prime}\right\rangle\right\} .
\end{aligned}
$$

Our first statement gives another representation of these functions, connecting them with the expansion factors defined by

$$
\begin{aligned}
& \rho^{+}(v)(x):=\sup \left\{\rho>0: x-\rho(\hat{F}(v)-v)^{*} \subset \Omega\right\} \quad \text { and } \\
& \rho^{-}(v)(x):=-\sup \left\{\rho>0: x+\rho(\hat{F}(v)-v)^{*} \subset \Omega\right\}
\end{aligned}
$$

for $v \in$ int $\hat{F}(v)$ and $x \in \Omega$; and $\rho^{+}(v)(x)=\rho^{-}(v)(x)=0$ for $x \in \partial \Omega$ or $v \in \partial \hat{F}(v)$.

Proposition 2.1. For all $v \in \operatorname{dom}\left(g^{* *}\right)$ and $x \in \bar{\Omega}$ we have

$$
s^{+}(v)(x)=\langle v, x\rangle+\rho^{+}(v)(x) \text { and } s^{-}(v)(x)=\langle v, x\rangle+\rho^{-}(v)(x) .
$$

If $u(\cdot)$ is a minimizer of the problem $\left(\mathscr{R} \mathscr{P}_{v}\right)$ for some $v \in \operatorname{dom}\left(g^{* *}\right)$ then

$$
s^{-}(v)(x) \leqslant u(x) \leqslant s^{+}(v)(x) \quad \forall x \in \bar{\Omega} .
$$

Proof. Let us prove, for instance, the first equality in (2.6). Set

$$
\Delta:=s^{+}(v)(x)-\langle v, x\rangle=\inf _{x^{\prime} \in \partial \Omega} \sigma_{\hat{F}(v)-v}\left(x-x^{\prime}\right) .
$$


If $v \in \partial \hat{F}(v)$ then there exists $\xi \in \mathbb{R}^{n}, \xi \neq 0$, with $\sigma_{\hat{F}(v)-v}(\xi)=0$. On the other hand, for each $x \in \Omega$ one can find $x^{\prime} \in \partial \Omega$ and $\lambda>0$ such that $x-x^{\prime}=\lambda \xi$. Therefore $\Delta=0=\rho^{+}(v)(x)$. Observe that the relation $v \in \partial \hat{F}(v)$ is equivalent to $\operatorname{int} \hat{F}(v)=\emptyset$.

Now assume that $v \in \operatorname{int} \hat{F}(v)$, i.e. the set $(\hat{F}(v)-v)^{*}$ is bounded. For an arbitrary $\varepsilon>0$ let us choose $x^{\prime} \in \partial \Omega$ such that $\sigma_{\hat{F}(v)-v}\left(x-x^{\prime}\right) \leqslant \Delta+\varepsilon$. It is equivalent to say that $x^{\prime} \in x+(\Delta+\varepsilon)\left[-(\hat{F}(v)-v)^{*}\right]$. Consequently, $\Delta+\varepsilon \geqslant \rho^{+}(v)(x)$, i.e., the inequality $\langle v, x\rangle+\rho^{+}(v)(x) \leqslant s^{+}(v)(x)$ holds. Since the set $-(\hat{F}(v)-v)^{*}$ is compact, it is not possible that $x+\rho^{+}(v)(x)\left[-(\hat{F}(v)-v)^{*}\right] \subset \Omega$. Then there exists $x^{\prime} \in \partial \Omega$ with

$$
\frac{x-x^{\prime}}{\rho^{+}(v)(x)} \in(\hat{F}(v)-v)^{*}
$$

or, in other words, $\sigma_{\hat{F}(v)-v}\left(x-x^{\prime}\right) \leqslant \rho^{+}(v)(x)$, i.e. the converse inequality also follows. The proof of the second equality in (2.6) is similar.

Now let $u(\cdot)$ be a minimizer of the relaxed functional subject to the boundary condition $u(x)=\langle v, x\rangle$ whenever $x \in \partial \Omega$. By (2.1) and boundedness of $\hat{F}(v)$, the function $u(\cdot)$ is Lipschitz continuous. Therefore, using Lebourg's theorem [6, p. 41] and Theorem 2.5.1 [6, p. 63] for each $x \in \Omega$ and $x^{\prime} \in \partial \Omega$, one can find $v^{\prime} \in \hat{F}(v)$ such that $u(x)-\left\langle v, x^{\prime}\right\rangle=$ $u(x)-u\left(x^{\prime}\right)=\left\langle v^{\prime}, x-x^{\prime}\right\rangle$; and this immediately implies the inequalities (2.7).

Remark 2.1. From Proposition 2.1 it follows, in particular, that the problem $\left(\mathscr{R} \mathscr{P}_{v}\right)$ admits a unique (affine) solution if and only if $v \in \partial \hat{F}(v)$.

Observe that the function $\rho^{+}(v)(\cdot)$ is the viscosity solution of the Hamilton-Jacobi equation $\sigma_{(\hat{F}(v)-v)^{*}}(\nabla u(x))=1,\left.\quad u\right|_{\partial \Omega}=0$, as defined in [8]. See, for instance, [2] and the references there. In order to give a generalization of the McShane lemma on Lipschitz extensions, the authors in [2] also used the symmetrical function $\rho^{-}(v)(\cdot)$.

Theorem 2.1. Let $g: \mathbb{R}^{n} \rightarrow \mathbb{R} \cup\{+\infty\}$ be a lower semicontinuous proper function and fix any open bounded set $\Omega$ in $\mathbb{R}^{n}$. Then the above functions $s^{+}(v)(\cdot)$ and $s^{-}(v)(\cdot)$ are minimizers in the relaxed problem $\left(\mathscr{R} \mathscr{P}_{v}\right)$ for each $v \in \operatorname{dom}\left(g^{* *}\right)$. Moreover, the mappings $v \mapsto s^{+}(v)(\cdot)$ and $v \mapsto s^{-}(v)(\cdot)$ are Lipschitz continuous in the sense that the inequalities

$$
\begin{aligned}
& \left\|s^{+}\left(v_{1}\right)-s^{+}\left(v_{2}\right)\right\|_{\infty} \leqslant\|\Omega\|\left|v_{1}-v_{2}\right| \text { and } \\
& \left\|s^{-}\left(v_{1}\right)-s^{-}\left(v_{2}\right)\right\|_{\infty} \leqslant\|\Omega\|\left|v_{1}-v_{2}\right|
\end{aligned}
$$

hold for all $v_{1}, v_{2} \in \operatorname{dom}\left(g^{* *}\right)$ where $\|\Omega\|:=\max _{x \in \bar{\Omega}}|x|$.

Proof. Given $v \in \operatorname{dom}\left(g^{* *}\right)$ the set $\hat{F}(v)-v$ is bounded, and for any $x^{\prime} \in \partial \Omega$ the map $x \mapsto \sigma_{\hat{F}(v)-v}\left(x-x^{\prime}\right)$ is Lipschitz continuous with Lipschiz constant not depending on $x^{\prime}$. Hence it is differentiable (a.e.) and $\nabla \sigma_{\hat{F}(v)-v}\left(x-x^{\prime}\right) \in \operatorname{ext} \hat{F}(v)-v$ (see 
[17, Corollary 25.1.3, p. 243]). Then $s^{+}(v)(x)=\langle v, x\rangle+\inf _{x^{\prime} \in \partial \Omega} \sigma_{\hat{F}(v)-v}\left(x-x^{\prime}\right)$ is also Lipschitz and, by Theorem 2.8.6 [6] $\nabla s^{+}(v)(x) \in \hat{F}(v)$ for a.e. $x \in \Omega$, i.e. $s^{+}(v)(\cdot)$ is the solution of $\left(\mathscr{R} \mathscr{P}_{v}\right)$.

Let us prove the inequality (2.8). It is obvious in case either $\hat{F}\left(v_{1}\right)=\hat{F}\left(v_{2}\right)$ or both sets $\hat{F}\left(v_{1}\right)$ and $\hat{F}\left(v_{2}\right)$ have empty interiors. Assume, therefore, that $\hat{F}\left(v_{1}\right) \cap \hat{F}\left(v_{2}\right)=\emptyset$ and $v_{1} \in \operatorname{int} \hat{F}\left(v_{1}\right), v_{2} \in \operatorname{int} \hat{F}\left(v_{2}\right)$. All the other cases can be easily reduced to this one. Denote by $v_{1}^{\prime}$ and $v_{2}^{\prime}$ the points of the interval $\left(v_{1}, v_{2}\right)$ which belong to the boundaries of $\hat{F}\left(v_{1}\right)$ and $\hat{F}\left(v_{2}\right)$, respectively. By definition $\rho^{+}\left(v_{1}^{\prime}\right)=\rho^{+}\left(v_{2}^{\prime}\right) \equiv 0$. Furthermore, given $\varepsilon>0$ small enough we have that $\rho^{+}(v)(x) \leqslant \varepsilon \forall x \in \bar{\Omega}$ whenever either $v \in \operatorname{int} \hat{F}\left(v_{1}\right)$ with $\left|v-v_{1}^{\prime}\right| \leqslant \varepsilon /(3\|\Omega\|)$, or $v \in$ int $\hat{F}\left(v_{2}\right)$ with $\left|v-v_{2}^{\prime}\right| \leqslant \varepsilon /(3\|\Omega\|)$. Indeed, assuming that $\rho^{+}(v)(x)>\varepsilon$ for some $x \in \Omega$, in the first case, e.g., we would have $x+\varepsilon\left[-\left(\hat{F}\left(v_{1}\right)-v\right)^{*}\right] \subset$ $\Omega$, hence $\left(\hat{F}\left(v_{1}\right)-v\right)^{*} \subset(2\|\Omega\| / \varepsilon) \overline{\mathbf{B}}$ or $v+\varepsilon /(2\|\Omega\|) \overline{\mathbf{B}} \subset \hat{F}\left(v_{1}\right)$ which is impossible. Now for any $\varepsilon>0$ choose points $v_{1}^{\prime \prime} \in\left(v_{1}, v_{1}^{\prime}\right)$ and $v_{2}^{\prime \prime} \in\left(v_{2}^{\prime}, v_{2}\right)$ with $\left|v_{1}^{\prime}-v_{1}^{\prime \prime}\right| \leqslant \varepsilon /(3\|\Omega\|)$, $\left|v_{2}^{\prime}-v_{2}^{\prime \prime}\right| \leqslant \varepsilon /(3\|\Omega\|)$. Since $v_{1}^{\prime \prime} \in \operatorname{int} \hat{F}\left(v_{1}\right)=\operatorname{int} \hat{F}\left(v_{1}^{\prime \prime}\right)$ and $v_{2}^{\prime \prime} \in \operatorname{int} \hat{F}\left(v_{2}\right)=\operatorname{int} \hat{F}\left(v_{2}^{\prime \prime}\right)$ we can write

$$
\begin{aligned}
\left|s^{+}\left(v_{1}\right)(x)-s^{+}\left(v_{2}\right)(x)\right| \leqslant & \left|s^{+}\left(v_{1}\right)(x)-s^{+}\left(v_{1}^{\prime \prime}\right)(x)\right| \\
& +\left|\left\langle v_{1}^{\prime \prime}, x\right\rangle+\rho^{+}\left(v_{1}^{\prime \prime}\right)(x)-\left\langle v_{1}^{\prime}, x\right\rangle\right| \\
& +\left|\left\langle v_{1}^{\prime}, x\right\rangle-\left\langle v_{2}^{\prime}, x\right\rangle\right| \\
& +\left|\left\langle v_{2}^{\prime}, x\right\rangle-\left\langle v_{2}^{\prime \prime}, x\right\rangle-\rho^{+}\left(v_{2}^{\prime \prime}\right)(x)\right| \\
& +\left|s^{+}\left(v_{2}^{\prime \prime}\right)(x)-s^{+}\left(v_{2}\right)(x)\right| \\
\leqslant & \|\Omega\|\left|v_{1}-v_{1}^{\prime \prime}\right|+\|\Omega\|\left|v_{1}^{\prime \prime}-v_{1}^{\prime}\right| \\
& +\|\Omega\|\left|v_{1}^{\prime}-v_{2}^{\prime}\right|+\|\Omega\|\left|v_{2}^{\prime}-v_{2}^{\prime \prime}\right| \\
& +\|\Omega\|\left|v_{2}^{\prime \prime}-v_{2}\right|+2 \varepsilon \\
= & \|\Omega\|\left|v_{1}-v_{2}\right|+2 \varepsilon .
\end{aligned}
$$

This proves (2.8). In a similar way one may consider the function $s^{-}(v)(x)$ and obtain the inequality (2.9).

Taking into account also Proposition 2.1 the functions $s^{+}(v)(\cdot)$ and $s^{-}(v)(\cdot)$ can be named the upper and lower solutions of the problem $\left(\mathscr{R} \mathscr{P}_{v}\right)$, respectively. Theorem 2.1 states their Lipschitz regularity w.r.t. the boundary data. Clearly, we can extend these functions to whole space $\mathbb{R}^{n}$ by setting $\rho^{+}(v)=\rho^{-}(v) \equiv 0 \quad \forall v \notin \operatorname{dom}\left(g^{* *}\right)$.

The following examples show that when some $n$-dimensional face of epi $\left(g^{* *}\right)$ is unbounded then the family of minimizers of the relaxed problem may be unbounded and/or may not contain its lower and upper bounds.

Example 2.1. Let $\Omega \subset \mathbb{R}^{n}$ be an open bounded set and $g(\xi)=1 /(|\xi|+1), \xi \in \mathbb{R}^{n}$.

Clearly, here $g^{* *}(\xi) \equiv 0$ and for each $v \in \mathbb{R}^{n}$ we have $\hat{F}(v)=\mathbb{R}^{n}$. Then any function from $\langle v, \cdot\rangle+\mathbf{W}_{0}^{1, p}(\Omega)$ is solution of the relaxed problem, and from (2.4)-(2.6) we see that $s^{+}(v)(x) \equiv+\infty$ and $s^{-}(v)(x) \equiv-\infty$. 
Example 2.2. Let $\Omega=(-1,1) \subset \mathbb{R}^{1}$ and $g(\xi)=|\xi|\left(1+1 /\left(\xi^{2}+1\right)\right), \xi \in \mathbb{R}^{1}$.

In this case $g^{* *}(\xi)=|\xi|$, and the boundary of epi $\left(g^{* *}\right)$ consists of two one-dimensional faces whose projections into $\mathbb{R}^{1}$ are $(-\infty, 0]$ and $[0,+\infty)$. From (2.2) and (2.3) one easily finds

$$
s^{+}(v)(x)=\left\{\begin{array}{ll}
-v & \text { if } x=-1, \\
|v| & \text { if }-1<x<1, \\
v & \text { if } x=1 .
\end{array} s^{-}(v)(x)= \begin{cases}-v & \text { if } x=-1, \\
-|v| & \text { if }-1<x<1, \\
v & \text { if } x=1 .\end{cases}\right.
$$

These functions are not even continuous in $x$, so that they are not minimizers in $\left(\mathscr{R}_{v}\right)$ for each $v \neq 0$. On the other hand, $s^{+}(v)(\cdot)$ and $s^{-}(v)(\cdot)$ are the upper and lower bounds of the solution set, respectively, in the sense that the problem $\left(\mathscr{R} \mathscr{P}_{v}\right)$ admits a (continuous in $v$ ) solution $s(v)(\cdot)$ which is so near to the function $s^{+}(v)(\cdot)$ (respectively, $\left.s^{-}(v)(\cdot)\right)$ as one wishes. For instance, let the continuous mappings $s_{k}: \mathbb{R}^{1} \rightarrow \mathbf{C}(\bar{\Omega})$ be defined as

$$
s_{k}(v)(x):= \begin{cases}k(v+|v|)(x+1)-v & \text { if }-1 \leqslant x<-1+1 / k, \\ |v| & \text { if }-1+1 / k \leqslant x \leqslant 1-1 / k, \\ k(v-|v|)(x-1)+v & \text { if } 1-1 / k<x \leqslant 1 .\end{cases}
$$

Then $s_{k}(v)(\cdot)$ is a minimizer of the relaxed functional for each $v \in \mathbb{R}^{1}, k=1,2, \ldots$, and the sequence $\left\{s_{k}(v)(\cdot)\right\}$ converges to $s^{+}(v)(\cdot)$ (but only pointwise).

\section{The main result: basic constructions}

The basic space in our considerations will be the space $\mathscr{C}\left(\mathbb{R}^{n}, \mathbf{C}(\bar{\Omega})\right)$ of all the continuous mappings $s: \mathbb{R}^{n} \rightarrow \mathbf{C}(\bar{\Omega})$ with the topology of uniform convergence in compact sets. This space is, clearly, metrizable and complete. Let us denote by $\mathscr{S}^{* *}$ the set of all $\rho(\cdot) \in$ $\mathscr{C}\left(\mathbb{R}^{n}, \mathbf{C}_{0}(\bar{\Omega})\right)$ such that the function $x \mapsto\langle v, x\rangle+\rho(v)(x)$ is solution of the relaxed problem $\left(\mathscr{R} \mathscr{P}_{v}\right)$ for all $v \in \operatorname{dom}\left(g^{* *}\right)$, and $\rho(v) \equiv 0$ if $v \notin \operatorname{dom}\left(g^{* *}\right)$. Observe that each function $\rho(\cdot) \in \mathscr{S}^{* *}$ vanishes outside the open set $\bigcup_{v \in \operatorname{dom}\left(g^{* *}\right)}$ int $\hat{F}(v)$ (see the Remark after Proposition 2.1). Since for different $v$ the sets int $\hat{F}(v)$ are either disjoint or coincide, this union consists of an at most countable family of disjoint sets, say $V_{m}, m=1,2, \ldots$, which are bounded under any of our hypotheses on a behaviour of $g$ at infinity. Recalling now the condition (2.1) we see that the set $\mathscr{S}^{* *}$ consists of those and only those continuous mappings $\rho: \mathbb{R}^{n} \rightarrow \mathbf{C}_{0}(\bar{\Omega})$ which are equal to zero outside $\bigcup_{m=1}^{\infty} V_{m}$ such that $\rho(v)(\cdot) \in$ $\mathbf{W}_{0}^{1, p}(\Omega)$ and $v+\nabla \rho(v)(x) \in \bar{V}_{m}$ for a.e. $x \in \Omega$ and all $v \in V_{m}, m=1,2, \ldots$. For each $\rho(\cdot) \in \mathscr{S}^{* *}$ in fact $\rho(v)(\cdot) \in \mathbf{W}_{0}^{1, \infty}(\Omega), v \in V_{m}$, and the function $\rho(v)(\cdot)$ is Lipschitz continuous with Lipschitz constant $D_{m}:=\operatorname{diam} V_{m}, m=1,2, \ldots$.

Lemma 3.1. The set $\mathscr{S}^{* *}$ is closed in $\mathscr{C}\left(\mathbb{R}^{n}, \mathbf{C}(\bar{\Omega})\right)$, hence it is a complete metric space with respect to the induced topology.

Proof. Let $\left\{\rho_{k}(\cdot)\right\}$ be a sequence in $\mathscr{S}^{* *}$ converging to some $\rho(\cdot) \in \mathscr{C}\left(\mathbb{R}^{n}, \mathbf{C}(\bar{\Omega})\right)$. We clearly have that $\rho(v)=0$ for all $v \notin \bigcup_{m=1}^{\infty} V_{m}$, and $\rho(v)(x)=0$ whenever $x \in \partial \Omega, v \in$ $\operatorname{dom}\left(g^{* *}\right)$. Furthermore, given $v \in V_{m}, m=1,2, \ldots$, we see that the sequence $\left\{\rho_{k}(v)(\cdot)\right\}$ is 
bounded in the space $\mathbf{W}^{1, \infty}(\Omega)$. Consequently, by the Alaoglu-Bourbaki theorem (see, e.g., $\left[19\right.$, p. 89]) we can assume without loss of generality that $\left\{\rho_{k}(v)(\cdot)\right\}$ converges to $\rho(v)(\cdot)$ weakly in $\mathbf{W}^{1, \infty}(\Omega)$. Therefore, the sequence of gradients $\left\{v+\nabla \rho_{k}(v)(\cdot)\right\}$ converges weakly in $\mathbf{L}^{\infty}(\Omega)$ to $v+\nabla \rho(v)(\cdot)$, and the relations $v+\nabla \rho_{k}(v)(x) \in \bar{V}_{m}, k=1,2, \ldots$, that hold for a.e. $x \in \Omega$, imply by convexity of $V_{m}$ that also $v+\nabla \rho(v)(x) \in \overline{\mathbf{V}}_{m}$ a.e. in $\Omega$. Thus $\rho(\cdot) \in \mathscr{S}^{* *}$ and the proof is complete.

From now on we consider $\mathscr{S}^{* *}$ as a metric space with the induced topology.

Let us pass to the original nonconvex variational problem $\left(\mathscr{P}_{v}\right)$ and observe (see [20, Lemma 2.3]) that each solution of $\left(\mathscr{P}_{v}\right)$ is also solution of the relaxed problem $\left(\mathscr{R} \mathscr{P}_{v}\right)$. So that it is natural to consider the set $\mathscr{S}$ of all the mappings $\rho(\cdot) \in \mathscr{S}^{* *}$ such that $x \mapsto$ $\langle v, x\rangle+\rho(v)(x)$ is solution of $\left(\mathscr{P}_{v}\right)$ for all $v \in \bigcup_{m=1}^{\infty} V_{m}$, taking into account that for other $v$ solution may fail to exist. On the other hand, we pay special attention to those elements $\rho(\cdot) \in \mathscr{S}^{* *}$ whose gradients $\nabla \rho(v)(x)$ belong to ext $V_{m}-v$ a.e. in $\Omega$ whenever $v \in V_{m}$, $m=1,2, \ldots$. As is easily seen, the set of all such mappings, say $\mathscr{S}^{\text {ext }}$, is contained in $\mathscr{S}$.

The main result of this paper is the following

Theorem 3.1. $\mathscr{S}^{\mathrm{ext}}$ is a dense $G_{\delta^{-}}$subset of $\mathscr{S}^{* *}$.

Thus $\mathscr{S}$ is also dense in $\mathscr{S}^{* *}$ which means that each solution of $\left(\mathscr{R}_{\mathscr{P}_{v}}\right)$ continuously depending on $v$ can be approximated by a sequence of solutions of the original problem $\left(\mathscr{P}_{v}\right)$ keeping continuity in $v$ uniformly in each bounded set where a solution exists, particularly, in each $V_{m}, m=1,2, \ldots$.

Below we introduce some constructions which will be used to prove this result.

Let us associate to each compact convex set $K \subset \mathbb{R}^{n}$ a function $l(\cdot, K): \mathbb{R}^{n} \rightarrow \overline{\mathbb{R}}$ concave, upper semicontinuous and such that

$$
\begin{aligned}
& 0 \leqslant l(x, K) \leqslant \operatorname{diam} K \quad \forall x \in K \\
& l(x, K)=-\infty \quad \forall x \notin K ; \\
& l(x, K)=0 \quad \text { if and only if } \quad x \in \operatorname{ext} K .
\end{aligned}
$$

We refer to [1,11] for examples of such functions. For fixed $m$ and $u(\cdot) \in \mathbf{C}(\bar{\Omega})$ we denote by

$$
L_{m}(u)=\int_{\Omega} l\left(\nabla u(x), \bar{V}_{m}\right) \mathrm{d} x
$$

It is known (see [9, p. 49]) that the functional $L_{m}: \mathbf{C}(\bar{\Omega}) \rightarrow \overline{\mathbb{R}}$ is upper semicontinuous.

Given $m=1,2, \ldots$ and $\eta>0$ small enough we consider then the compact set

$$
V_{m}^{\eta}:=\left\{v \in V_{m}: \operatorname{dist}_{\partial V_{m}}(v) \geqslant \eta\right\}
$$

and for each $\rho(\cdot) \in \mathscr{S}^{* *}$, we define

$$
\mathscr{L}_{m}^{\eta}(\rho):=\sup _{v \in V_{m}^{\eta}} L_{m}(\langle v, \cdot\rangle+\rho(v)(\cdot)) .
$$


Lemma 3.2. The functional $\mathscr{L}_{m}^{\eta}: \mathscr{S}^{* *} \rightarrow \mathbb{R}$ is bounded, namely

$$
0 \leqslant \mathscr{L}_{m}^{\eta}(\rho) \leqslant D_{m} \cdot \mu(\Omega) \quad \forall \rho(\cdot) \in \mathscr{S}^{* *},
$$

and is upper semicontinuous.

Proof. Since for $\rho(\cdot) \in \mathscr{S}^{* *}$ we have $v+\nabla \rho(v)(x) \in \bar{V}_{m}$ a.e. in $\Omega$ whenever $v \in V_{m}$, the inequality (3.6) follows immediately from (3.5), (3.3) and (3.1).

Let now $\left\{\rho_{k}\right\} \subset \mathscr{S}^{* *}$ be a sequence converging to $\rho(\cdot)$ in $\mathscr{S}^{* *}$. For each $k=1,2, \ldots$ let us choose $v_{k} \in V_{m}^{\eta}$ such that

$$
\mathscr{L}_{m}^{\eta}\left(\rho_{k}\right)=L_{m}\left(\left\langle v_{k}, \cdot\right\rangle+\rho_{k}\left(v_{k}\right)(\cdot)\right) .
$$

The existence of such $v_{k}$ follows from upper semicontinuity of the mapping $v \mapsto L_{m}(\langle v, \cdot\rangle+$ $\left.\rho_{k}(v)(\cdot)\right)$ and compactness of $V_{m}^{\eta}$. Without loss of generality we can assume that the sequence $\left\{v_{k}\right\}$ converges to some point $v \in V_{m}^{\eta}$. Then, obviously, $\rho_{k}\left(v_{k}\right) \rightarrow \rho(v)$ as $k \rightarrow \infty$ in $\mathbf{C}(\bar{\Omega})$, and by the same argument as above

$$
L_{m}(\langle v, \cdot\rangle+\rho(v)(\cdot)) \geqslant \underset{k \rightarrow \infty}{\lim \sup _{m}} L_{m}\left(\left\langle v_{k}, \cdot\right\rangle+\rho_{k}\left(v_{k}\right)(\cdot)\right) .
$$

Thus, from (3.8), (3.5) and (3.7) we obtain

$$
\limsup _{k \rightarrow \infty} \mathscr{L}_{m}^{\eta}\left(\rho_{k}\right) \leqslant \mathscr{L}_{m}^{\eta}(\rho),
$$

and the upper semicontinuity of $\mathscr{L}_{m}^{\eta}$ follows.

We associate to the functional $\mathscr{L}_{m}^{\eta}$ the sublevel set

$$
\mathscr{S}_{m}^{\eta}:=\left\{\rho(\cdot) \in \mathscr{S}^{* *}: \mathscr{L}_{m}^{\eta}(\rho)<\eta\right\}
$$

which is open by Lemma 3.2. Taking into account the property (3.2) we see that

$$
\bigcap_{m, k=1}^{\infty} \mathscr{S}_{m}^{1 / k}=\mathscr{S}^{\mathrm{ext}}
$$

Therefore, in order to apply the Baire category theorem to prove density of the set $\mathscr{S}^{\text {ext }}$ in $\mathscr{S}^{* *}$ it suffices to prove density of each $\mathscr{S}_{m}^{\eta}, m=1,2, \ldots, \eta>0$. We deal with this in Section 5. In the next section, instead, we obtain some auxiliary approximation results.

In what follows we fix $m \in\{1,2, \ldots\}$ and, for the sake of simplicity, use the notations $V, D, V^{\eta}$ and $\mathscr{S}^{\eta}$ in the place of $V_{m}, D_{m}, V_{m}^{\eta}$ and $\mathscr{S}_{m}^{\eta}$, respectively.

\section{The polynomial approximations}

Let us denote by $\mathscr{C}_{V}$ the family of continuous mappings $\theta: \bar{V} \rightarrow \mathbf{C}(\bar{\Omega})$ such that $\nabla \theta(v)(x) \in \bar{V}-v$ a.e. in $\Omega$ for all $v \in V$. Observe that the restriction of the set $\mathscr{S}^{* *}$ to $\bar{V}$ consists of all $\theta(\cdot) \in \mathscr{C}_{V}$ satisfying the suplementary condition $\theta(v)(x)=0 \forall(v, x) \in$ $(\bar{V} \times \partial \Omega) \cup(\partial V \times \bar{\Omega})$. We denote this set by $\mathscr{S}_{V}^{* *}$. 
Lemma 4.1. Let $\theta(\cdot) \in \mathscr{C}_{V}$ be such that the function $(v, x) \mapsto \theta(v)(x)$ is constant on the set $\bar{V} \times \partial \Omega$. Then there exists a sequence $\left\{\theta_{k}(\cdot)\right\} \subset \mathscr{C}_{V}$ converging to $\theta(\cdot)$ as $k \rightarrow \infty$ uniformly in $v \in \bar{V}$, such that for each $v \in V$ and $k \in\{1,2, \ldots\}$ either $\theta_{k}(v) \equiv 0$ or $\theta_{k}(v)(x) \neq 0$ for a.e. $x \in \Omega$.

Proof. Assume that $\theta(v)(x)=\alpha=$ const $\forall v \in \bar{V}, x \in \partial \Omega$, and extend each function $\theta(v)(\cdot), v \in \bar{V}$, to $\mathbb{R}^{n}$ setting $\theta(v)(x)=\alpha$ for $x \notin \bar{\Omega}$. We apply then the standard argument of polynomial approximations (see, e.g., [18, p. 159]).

Fix $R \geqslant \frac{3}{4}$ so large that $\bar{\Omega}-\bar{\Omega} \subset \Pi_{R}$ where

$$
\Pi_{R}:=\left\{x \in \mathbb{R}^{n}:\left|x_{i}\right| \leqslant R \forall i=1, \ldots, n\right\} .
$$

Let us define the polynomials

$$
Q_{k}(x):=\gamma_{k} \prod_{i=1}^{n}\left(R^{2}-x_{i}^{2}\right)^{k}, \quad x=\left(x_{1}, \ldots, x_{n}\right) \in \mathbb{R}^{n}, \quad k=1,2, \ldots,
$$

where the constants $\gamma_{k}>0$ are chosen such that

$$
\int_{\Pi_{R}} Q_{k}(x) \mathrm{d} x=1 .
$$

From the condition (4.1) we have

$$
\begin{aligned}
1 & =\gamma_{k} 2^{n}\left(\int_{0}^{R}\left(R^{2}-t^{2}\right) \mathrm{d} t\right)^{n} \\
& \geqslant \gamma_{k} 2^{n} R^{2 k n}\left(\int_{0}^{R / \sqrt{k}}\left(1-\frac{k t^{2}}{R^{2}}\right) \mathrm{d} t\right)^{n}=\left(\frac{4}{3}\right)^{n} \gamma_{k} R^{2 k n} R^{n}(1 / \sqrt{k})^{n} .
\end{aligned}
$$

Thus

$$
\gamma_{k} \leqslant \frac{(\sqrt{k})^{n}}{R^{2 k n}}, \quad k=1,2, \ldots
$$

Now given some $0<\delta<R$ for each $x \in \Pi_{R} \backslash \Pi_{\delta}$ we obtain

$$
0 \leqslant Q_{k}(x) \leqslant \gamma_{k}\left(R^{2}-\delta^{2}\right)^{k} R^{2 k(n-1)} \leqslant(\sqrt{k})^{n}\left(1-(\delta / R)^{2}\right)^{k}, \quad k=1,2, \ldots .
$$

Hence $\left\{Q_{k}(x)\right\}$ converges to zero as $k \rightarrow \infty$ uniformly in $x \in \Pi_{R} \backslash \Pi_{\delta}$. We set

$$
\theta_{k}(v)(x):=\int_{\Pi_{R}} \theta(v)(x+y) Q_{k}(y) \mathrm{d} y, \quad x \in \bar{\Omega}, \quad v \in \bar{V},
$$

and observe that the mappings $\theta_{k}: \bar{V} \rightarrow \mathbf{C}(\bar{\Omega})$ are continuous, and

$$
\nabla \theta_{k}(v)(x)=\int_{\Pi_{R}} \nabla \theta(v)(x+y) Q_{k}(y) \mathrm{d} y \in \bar{V}-v
$$


a.e. in $\Omega$ whenever $v \in V$, that is $\theta_{k}(\cdot) \in \mathscr{C}_{V}$. Furthermore, given $\varepsilon>0$, by continuity of the mapping $(x, v) \mapsto \theta(v)(x)$ and compactness of the sets $\bar{\Omega}, \bar{V}$ one can choose $\delta>0$ such that

$$
|\theta(v)(x+y)-\theta(v)(x)| \leqslant \varepsilon / 2,
$$

whenever $v \in \bar{V}, x \in \mathbb{R}^{n}$ and $y \in \Pi_{\delta}$. Also there exists $L>0$ such that $|\theta(v)(x)| \leqslant L$ for all $v \in \bar{V}$ and $x \in \mathbb{R}^{n}$. By uniform convergence of the polynomials $Q_{k}(x)$, let us choose $k^{\prime} \in\{1,2, \ldots\}$ such that

$$
0 \leqslant Q_{k}(x) \leqslant \frac{\varepsilon}{2 L(2 R)^{n}}
$$

for all $k \geqslant k^{\prime}$ and all $x \in \Pi_{R} \backslash \Pi_{\delta}$. Combining (4.2) and (4.3) we obtain

$$
\begin{aligned}
\left|\theta_{k}(v)(x)-\theta(v)(x)\right| \leqslant & \int_{\Pi_{\delta}}|\theta(v)(x+y)-\theta(v)(x)| Q_{k}(y) \mathrm{d} y \\
& +\int_{\Pi_{R} \backslash \Pi_{\delta}}|\theta(v)(x+y)-\theta(v)(x)| Q_{k}(y) \mathrm{d} y \\
\leqslant & \varepsilon / 2+2 L \int_{\Pi_{R} \backslash \Pi_{\delta}} Q_{k}(y) \mathrm{d} y \leqslant \varepsilon,
\end{aligned}
$$

whenever $v \in \bar{V}$ and $x \in \bar{\Omega}$.

Finally, by a change of variables we have

$$
\begin{aligned}
\theta_{k}(v)(x)-\alpha & =\int_{\Pi_{R}}(\theta(v)(x+y)-\alpha) Q_{k}(y) \mathrm{d} y \\
& =\int_{\Pi_{R}+x}(\theta(v)(z)-\alpha) Q_{k}(z-x) \mathrm{d} z \\
& =\int_{\Omega}(\theta(v)(z)-\alpha) Q_{k}(z-x) \mathrm{d} z, \quad x \in \bar{\Omega}, \quad v \in \bar{V} .
\end{aligned}
$$

Therefore, $\theta_{k}(v)(\cdot)$ is polynomial of degree $2 n k$ w.r.t. $x_{i}, i=1, \ldots, n$, whenever $v \in \bar{V}$, and there are only two possibilities: either $\theta_{k}(v)(x) \neq 0$ for a.e. $x \in \Omega$ or $\theta_{k}(v) \equiv 0$ in $\Omega$ (see, e.g., [13, p. 120]).

Remark 4.1. If, in addition to the hypotheses of Lemma 4.1, we assume that $\theta(v)$ is not identically zero for each $v \in \bar{V}$, then by uniform convergence of the sequence $\left\{\theta_{k}(\cdot)\right\}$ it follows that for $k$ large enough we cannot have $\theta_{k}(x) \equiv 0$ for any $v \in \bar{V}$.

Corollary 4.1. For each $\tilde{\theta}(\cdot) \in \mathscr{S}_{V}^{* *}$ and $\varepsilon>0$ there exists $\bar{\theta}(\cdot) \in \mathscr{C}_{V}$ such that, for any $v \in \bar{V}$

$$
\|\tilde{\theta}(v)-\bar{\theta}(v)\|_{\infty} \leqslant \varepsilon
$$

and

$$
\bar{\theta}(v)(x) \neq 0 \quad \text { for a.e. } x \in \Omega \text {. }
$$


Proof. It is enough to apply Lemma 4.1 and the Remark above to the function $\theta(v)=$ $\tilde{\theta}(v)+\varepsilon / 2$.

The approximating function $\bar{\theta}(\cdot)$ constructed in Corollary 4.1 keeps the property of gradients to stay inside the convex set $\bar{V}-v$ but it no more vanishes on the boundaries $\partial \Omega$ and $\partial V$. In order to construct a function satisfying both the boundary condition and the property (4.5) let us use the upper and lower solutions of the problem $(\mathscr{R} \mathscr{P} v)$ considered in Section 2.

Proposition 4.1. Given any $\tilde{\rho}(\cdot) \in \mathscr{S}^{* *}$ and $\varepsilon>0$ there exists a $\bar{\rho}(\cdot) \in \mathscr{S}^{* *}$ such that

$$
\|\bar{\rho}(v)-\tilde{\rho}(v)\|_{\infty} \leqslant \varepsilon \quad \forall v \in \mathbb{R}^{n}
$$

and, for any $v \in V$,

$$
\bar{\rho}(v)(x) \neq 0 \quad \text { for a.e. } x \in \Omega \text {. }
$$

Proof. Let $\bar{\theta}(\cdot) \in \mathscr{C}_{V}$ be the mapping obtained in Corollary 4.1 by assuming $\tilde{\theta}(\cdot)$ to be the restriction of $\tilde{\rho}(\cdot)$ to $\bar{V}$. We set

$$
\begin{aligned}
& \bar{\rho}^{+}(v)(x):=\min \left\{\max \{\bar{\theta}(v)(x), 0\}, \rho^{+}(v)(x)\right\}, \\
& \bar{\rho}^{-}(v)(x):=\max \left\{\min \{\bar{\theta}(v)(x), 0\}, \rho^{-}(v)(x)\right\},
\end{aligned}
$$

where $\rho^{+}$and $\rho^{-}$are defined by (2.4) and (2.5). Clearly, $\bar{\rho}^{+}$and $\bar{\rho}^{-}$are continuous mappings from $\bar{V}$ to $\mathbf{C}_{0}(\bar{\Omega})$. Furthermore, $\bar{\rho}^{+}(v)=\bar{\rho}^{-}(v) \equiv 0$ if $v \in \partial V$, and for each $v \in V$ the functions $\bar{\rho}^{+}(v)(\cdot), \bar{\rho}^{-}(v)(\cdot)$ are Lipschitz continuous. Since by Theorem 2.1, together with Proposition 2.1, the gradients $\nabla \rho^{+}(v)(x)$ and $\nabla \rho^{-}(v)(x)$ belong to the set $\bar{V}-v$ a.e. in $\Omega$ for all $v \in V$ and the same holds for the gradients $\nabla \bar{\theta}(v)(x)$ (see Corollary 4.1), then as it follows from Proposition 2.4 [12]

$$
\nabla \bar{\rho}^{+}(v)(x) \in \bar{V}-v, \quad \nabla \bar{\rho}^{-}(v)(x) \in \bar{V}-v
$$

for a.e. $x \in \Omega$ and all $v \in V$.

Let us define now

$$
\bar{\rho}(v)(x)=\bar{\rho}^{+}(v)(x)+\bar{\rho}^{-}(v)(x), \quad x \in \bar{\Omega}, \quad v \in \bar{V},
$$

and extend the mapping $\bar{\rho}: \bar{V} \rightarrow \mathbf{C}_{0}(\bar{\Omega})$ to $\mathbb{R}^{n}$ by setting $\bar{\rho}(v)=\tilde{\rho}(v)$ outside $\bar{V}$. So that $\bar{\rho}(\cdot) \in \mathscr{C}\left(\mathbb{R}^{n}, \mathbf{C}_{0}(\bar{\Omega})\right)$. If $\bar{\rho}^{+}(v)(x)>0$ for some $x \in \Omega, v \in V$ then $\bar{\theta}(v)(x)>0$ and $\bar{\rho}^{-}(v)(x)=0$ (we recall that $\rho^{+}(v)(x)>0$ and $\rho^{-}(v)(x)<0 \forall x \in \Omega, \quad v \in V$ ). Analogously, the inequality $\bar{\rho}^{-}(v)(x)<0$ implies $\bar{\rho}^{+}(v)(x)=0$. Therefore, $\bar{\rho}(v)(x)=0$ iff $\bar{\rho}^{+}(v)(x)=\bar{\rho}^{-}(v)(x)=0$, and in case $v \in V$ it follows that $\bar{\rho}(v)(x) \neq 0$ a.e. in $\Omega$. So that the property (4.6) holds. Furthermore, since for each $v \in V$ the function $\bar{\rho}(v)(x)$ is equal to either $\bar{\rho}^{+}(v)(x)$ or $\bar{\rho}^{-}(v)(x)$ on open subsets of $\Omega$ we have from (4.7) that $\bar{\rho}(\cdot) \in \mathscr{S}^{* *}$.

Finally, we have to show that

$$
|\tilde{\rho}(v)(x)-\bar{\rho}(v)(x)| \leqslant \varepsilon
$$


for all $v \in V, x \in \Omega$. This is obvious if $\bar{\rho}(v)(x)=0$ (see (4.4)). Consider now, for instance, the case $\bar{\rho}^{+}(v)(x)>0$. We have two possibilities. The first one is $\bar{\rho}(v)(x)=$ $\bar{\rho}^{+}(v)(x)=\bar{\theta}(v)(x)$, and the inequality (4.8) follows directly from (4.4). Otherwise, we have $\bar{\rho}(v)(x)=\bar{\rho}^{+}(v)(x)=\rho^{+}(v)(x)<\bar{\theta}(v)(x)$. Taking into account Proposition 2.1 we observe that $\tilde{\rho}(v)(x) \leqslant \rho^{+}(v)(x)$, and, therefore, $|\tilde{\rho}(v)(x)-\bar{\rho}(v)(x)| \leqslant|\tilde{\rho}(v)(x)-\bar{\theta}(v)(x)| \leqslant \varepsilon$. The case $\bar{\rho}^{-}(v)(x)<0$ can be treated similarly.

\section{The proof of density of $\mathscr{S}^{\eta}$ in $\mathscr{S}^{* *}$}

As proved in the previous section, each mapping $\tilde{\rho}(\cdot) \in \mathscr{S}^{* *}$ can be approximated uniformly by another one also belonging to $\mathscr{S}^{* *}$ and satisfying (4.6). Furthermore, the last condition implies the following property which is crucial to prove the main theorem.

Lemma 5.1. Assume that the mapping $\bar{\rho}(\cdot) \in \mathscr{C}\left(\mathbb{R}^{n}, \mathbf{C}(\bar{\Omega})\right)$ satisfies the condition (4.6). Then for each compact $K \subset V$ and $\zeta>0$ there exists $\delta>0$ such that

$$
\mu\{x \in \Omega:|\bar{\rho}(v)(x)|<\delta\}<\zeta \quad \forall v \in K .
$$

Proof. Indeed, by (4.6) to each $v \in V$ we can associate a number $\delta_{v}>0$ with

$$
\mu\left\{x \in \Omega:|\bar{\rho}(v)(x)|<\delta_{v}\right\}<\zeta .
$$

Let us choose then $\beta_{v}>0$ such that $\left|v^{\prime}-v\right| \leqslant \beta_{v}$ implies $\left\|\bar{\rho}\left(v^{\prime}\right)-\bar{\rho}(v)\right\|_{\infty} \leqslant \delta_{v} / 2$. Since the set $K \subset V$ is compact, we can find a finite number of points $v_{1}, \ldots, v_{r} \in K$ such that $K \subset \bigcup_{i=1}^{r}\left(v_{i}+\beta_{v_{i}} \overline{\mathbf{B}}\right)$. Now we set $\delta:=\min \left\{\delta_{v_{i}} / 2: i=1, \ldots, r\right\}$. Let us take any $v \in K$. Then there exists $v_{i}, i=1, \ldots, r$, with $\left|v-v_{i}\right| \leqslant \beta_{v_{i}}$, and, consequently, the inequality $\left|\bar{\rho}(v)(x)-\bar{\rho}\left(v_{i}\right)(x)\right| \leqslant \delta_{v_{i}} / 2$ holds for all $x \in \Omega$. Therefore, $|\bar{\rho}(v)(x)|<\delta \leqslant \delta_{v_{i}} / 2$ implies $\left|\bar{\rho}\left(v_{i}\right)(x)\right| \leqslant \delta_{v_{i}}$, and (5.1) follows from (5.2).

Proof of Theorem 3.1. Fix $\eta>0, \tilde{\rho}(\cdot) \in \mathscr{S}^{* *}$ and $\varepsilon>0$. By Proposition 4.1 there exists a mapping $\bar{\rho}(\cdot) \in \mathscr{S}^{* *}$ satisfying the condition (4.6) and with $\|\bar{\rho}(v)-\tilde{\rho}(v)\|_{\infty} \leqslant \varepsilon / 2$ $\forall v \in \mathbb{R}^{n}$. Let us choose, moreover, a number $0<\delta<\varepsilon / 2$ as in Lemma 5.1 for $K=V^{\eta}$ and $\zeta=\eta / D$ (see (3.4) and (3.6)).

We associate to each $v \in V$ the sets

$$
\begin{aligned}
& \Omega_{\delta}^{+}(v):=\{x \in \bar{\Omega}: \bar{\rho}(v)(x) \geqslant \delta\}, \\
& \Omega_{\delta}^{-}(v):=\{x \in \bar{\Omega}: \bar{\rho}(v)(x) \leqslant-\delta\}
\end{aligned}
$$

and observe that $\Omega_{\delta}^{+}$and $\Omega_{\delta}^{-}$have closed graphs, as multivalued functions from $V$ to $\bar{\Omega}$. Hence the sets

$$
\Gamma_{\delta}^{+}:=\left\{(v, x) \in V^{\eta} \times \bar{\Omega}: x \in \Omega_{\delta}^{+}(v)\right\}
$$

and

$$
\Gamma_{\delta}^{-}:=\left\{(v, x) \in V^{\eta} \times \bar{\Omega}: x \in \Omega_{\delta}^{-}(v)\right\}
$$


are compact. Consider, first, the covering of $\Gamma_{\delta}^{+}$by the family of open sets $U^{+}(x), x \in \Omega$, defined as

$$
\begin{aligned}
U^{+}(x):= & \{(v, y) \in V \times \Omega: \bar{\rho}(v)(x)>\delta / 2,|x-y|<\varepsilon /(8 D), \\
& \left.y \in x+(\bar{\rho}(v)(x)-\delta / 2) \operatorname{int}\left[-(V-v)^{*}\right] \subset \Omega\right\} .
\end{aligned}
$$

Notice that the inclusion $x+\bar{\rho}(v)(x) \operatorname{int}\left[-(V-v)^{*}\right] \subset \Omega$ always takes place due to the results of Section 2. By compactness there exists a finite number of points $x_{i}^{+}, i=1, \ldots, N^{+}$, in $\Omega$ such that

$$
\Gamma_{\delta}^{+} \subset \bigcup_{i=1}^{N^{+}} U^{+}\left(x_{i}^{+}\right) .
$$

Now we are able to define the function to be a suitable approximation of the positive part of $\bar{\rho}(v)(x)$, at least for $v \in V^{\eta}$. Namely, set

$$
f^{+}(v)(x):=\max _{1 \leqslant i \leqslant N^{+}}\left\{\bar{\rho}(v)\left(x_{i}^{+}\right)-\sigma_{V-v}\left(x_{i}^{+}-x\right), \delta / 2\right\}
$$

for $v \in \bar{V}, x \in \bar{\Omega}$. Obviously, $f^{+}: \bar{V} \rightarrow \mathbf{C}(\bar{\Omega})$ is a continuous mapping and $f^{+}(v)(x)=\delta / 2$ if $x \in \partial \Omega, v \in \bar{V}$, because in this case $\bar{\rho}(v)(x)=0$ and $\sigma_{V-v}\left(x_{i}^{+}-x\right) \geqslant \bar{\rho}(v)\left(x_{i}^{+}\right)$for all $i=1, \ldots, N^{+}$. Since for each $v \in V$ the function $f^{+}(v)(\cdot)$ is Lipschitz continuous, it admits gradient almost everywhere in $\Omega$ which is equal to either one of the gradients $\nabla \sigma_{V-v}\left(x_{i}^{+}-x\right), i=1, \ldots, N^{+}$, or zero (see [12, Proposition 2.4] or [16, p. 50]). So that we always have $\nabla f^{+}(v)(x) \in \bar{V}-v$ a.e. in $\Omega$.

Similarly, the open sets

$$
\begin{aligned}
U^{-}(x):= & \{(v, y) \in V \times \Omega: \bar{\rho}(v)(x)<-\delta / 2, \quad|x-y|<\varepsilon /(8 D), \\
& \left.y \in x-(\bar{\rho}(v)(x)+\delta / 2) \operatorname{int}(V-v)^{*} \subset \Omega\right\},
\end{aligned}
$$

$x \in \Omega$, cover $\Gamma_{\delta}^{-}$. Let $x_{i}^{-}, i=1, \ldots, N^{-}$, be some points of $\Omega$ such that

$$
\Gamma_{\delta}^{-} \subset \bigcup_{i=1}^{N^{-}} U^{-}\left(x_{i}^{-}\right),
$$

and $f^{-}: \bar{V} \rightarrow \mathbf{C}(\bar{\Omega})$ be the continuous mapping defined as

$$
f^{-}(v)(x):=\min _{1 \leqslant i \leqslant N^{-}}\left\{\bar{\rho}(v)\left(x_{i}^{-}\right)+\sigma_{V-v}\left(x-x_{i}^{-}\right),-\delta / 2\right\}
$$

for $v \in \bar{V}, x \in \bar{\Omega}$. By the same arguments as above it follows that $f^{-}(v)(x)=-\delta / 2$ whenever $x \in \partial \Omega, v \in \bar{V}$, and for each $v \in V$ the gradient $\nabla f^{-}(v)(x)$ belongs to $\bar{V}-v$ for a.e. $x \in \Omega$.

Fix $(v, x) \in \bar{V} \times \bar{\Omega}$. If $f^{+}(v)(x)>\delta / 2$ then for some $i=1, \ldots, N^{+}$and any $j=1, \ldots, N^{-}$ we have

$$
\begin{aligned}
\delta / 2 & <\bar{\rho}(v)\left(x_{i}^{+}\right)-\sigma_{V-v}\left(x_{i}^{+}-x\right) \\
& \leqslant \bar{\rho}(v)(x) \leqslant \bar{\rho}(v)\left(x_{j}^{-}\right)+\sigma_{V-v}\left(x-x_{j}^{-}\right),
\end{aligned}
$$


so that $f^{-}(v)(x)=-\delta / 2$. Analogously, assuming $f^{-}(v)(x)<-\delta / 2$ it follows immediately that $f^{+}(v)(x)=\delta / 2$. Thus, the function

$$
f(v)(x):=f^{+}(v)(x)+f^{-}(v)(x), \quad v \in \bar{V}, \quad x \in \bar{\Omega},
$$

is well defined in the sense that it is never equal to the sum of two nonconstant terms. Hence, as above (see the proof of Proposition 4.1) $\nabla f(v)(x) \in \bar{V}-v$ for a.e. $x \in \Omega$ and all $v \in \bar{V}$.

Let $v \in V^{\eta}$ and $x \in \Omega$ be such that the gradient $\nabla f(v)(x)$ exists. If $\bar{\rho}(v)(x) \geqslant \delta$ then by (5.4) and (5.3) there exists $i=1, \ldots, N^{+}$with

$$
x_{i}^{+}-x \in\left(\bar{\rho}(v)\left(x_{i}^{+}\right)-\delta / 2\right) \operatorname{int}(V-v)^{*},
$$

and this means

$$
\bar{\rho}(v)\left(x_{i}^{+}\right)-\sigma_{V-v}\left(x_{i}^{+}-x\right)>\delta / 2 .
$$

Consequently, according to Proposition 2.4 [12] and Corollary 25.1.3 [17, p. 243] it follows from (5.5) that $\nabla f(v)(x)=\nabla f^{+}(v)(x) \in$ ext $V-v$. Similarly, we have $v+\nabla f(v)(x) \in$ ext $V$ also for a.e. $x \in \Omega$ with $\bar{\rho}(v)(x) \leqslant-\delta$. By the choice of $\delta$ (see Lemma 5.1) we obtain

$$
\int_{\Omega} l(v+\nabla f(v)(x), \bar{V}) \mathrm{d} x \leqslant D \cdot \mu\{x \in \Omega:|\bar{\rho}(v)(x)|<\delta\}<\eta .
$$

Let us prove now that

$$
|f(v)(x)-\bar{\rho}(v)(x)|<\varepsilon / 2,
$$

whenever $v \in V^{\eta}, x \in \bar{\Omega}$. Fix $x \in \Omega$. As we already know, three cases here are possible.

Case $1 f^{+}(v)(x)>\delta / 2$.

By (5.8) we have $\bar{\rho}(v)(x)>\delta / 2$. If $\bar{\rho}(v)(x) \geqslant \delta$ then by (5.4) and (5.3) there exists $i=$ $1, \ldots, N^{+}$with $\bar{\rho}(v)\left(x_{i}^{+}\right)-\sigma_{V-v}\left(x_{i}^{+}-x\right)>\delta / 2$ and $\left|x-x_{i}^{+}\right|<\varepsilon /(8 D)$. Consequently (see $(5.5))$,

$$
\begin{aligned}
\bar{\rho}(v)(x) & >f(v)(x)=f^{+}(v)(x)-\delta / 2 \\
& \geqslant \bar{\rho}(v)\left(x_{i}^{+}\right)-\sigma_{V-v}\left(x_{i}^{+}-x\right)-\delta / 2 \\
& \geqslant \bar{\rho}(v)(x)-2 D\left|x-x_{i}^{+}\right|-\delta / 2>\bar{\rho}(v)(x)-\varepsilon / 2 .
\end{aligned}
$$

Otherwise we have $\delta / 2<\bar{\rho}(v)(x)<\delta$ hence

$$
\begin{aligned}
\bar{\rho}(v)(x) & >f(v)(x)=f^{+}(v)(x)-\delta / 2>0 \\
& >\bar{\rho}(v)(x)-\delta>\bar{\rho}(v)(x)-\varepsilon / 2
\end{aligned}
$$

and the inequality (5.10) also follows.

Case $2 f^{-}(v)(x)<-\delta / 2$.

This case is treated similarly.

Case $3 f^{+}(v)(x)=\delta / 2$ and $f^{-}(v)(x)=-\delta / 2$, so that $f(v)(x)=0$.

Assuming $\bar{\rho}(v)(x) \geqslant \delta$, like above we find $i=1, \ldots, N^{+}$with the property $\bar{\rho}(v)\left(x_{i}^{+}\right)-$ $\sigma_{V-v}\left(x_{i}^{+}-x\right)>\delta / 2$. However, this is not possible due to (5.5). Analogously, we always have $\bar{\rho}(v)(x)>-\delta$ and the inequality (5.10) holds. 
Since $V^{\eta}$ is compact, there exists an open set $W, V^{\eta} \subset W \subset V$, such that $\| f(v)-$ $\bar{\rho}(v) \|_{\infty}<\varepsilon / 2$ for all $v \in W$. Let $\psi: \mathbb{R}^{n} \rightarrow[0,1]$ be a continuous function equal to 1 on $V^{\eta}$ and to 0 outside $W$. Define the continuous mapping $\hat{\rho}: \mathbb{R}^{n} \rightarrow \mathbf{C}_{0}(\bar{\Omega})$ by

$$
\hat{\rho}(v)(x)=\psi(v) f(v)(x)+(1-\psi(v)) \bar{\rho}(v)(x), \quad v \in \mathbb{R}^{n}, \quad x \in \bar{\Omega},
$$

and observe that $\hat{\rho}(\cdot) \in \mathscr{S}^{* *}$. Since $\hat{\rho}(v)(x)=f(v)(x) \forall x \in \bar{\Omega}, v \in V^{\eta}$, it follows from (5.9) that $\hat{\rho}(\cdot) \in \mathscr{S}^{\eta}$. Finally, given an arbitrary $v \in \mathbb{R}^{n}$ we have

$$
\|\hat{\rho}(v)-\bar{\rho}(v)\|_{\infty} \leqslant \psi(v)\|f(v)-\bar{\rho}(v)\|_{\infty} \leqslant \varepsilon / 2,
$$

and Theorem 3.1 is completely proved.

Let us show now that if epi $\left(g^{* *}\right)$ has unbounded $n$-dimensional faces then the density property can be violated. This can be done through a slight modification of Example 2.2.

Example 5.1. Consider the lagrangian $g: \mathbb{R}^{1} \rightarrow \mathbb{R}^{1}$ defined by

$$
g(\xi):=|\xi|\left|1+\frac{|| \xi|-1|}{\xi^{2}+1}\right|
$$

and the domain $\Omega=(-1,1) \subset \mathbb{R}^{1}$.

Here, as happened in Example 2.2, the bipolar function is $g^{* *}(\xi)=|\xi|, \xi \in \mathbb{R}^{1}$; but in this case we have $g^{* *}( \pm 1)=g( \pm 1), g^{* *}(0)=g(0)$ while at other points $g^{* *}(\xi)<g(\xi)$. Consequently, by Theorem 1.1 [20], the problem $\left(\mathscr{P}_{v}\right)$ admits solutions iff $|v| \leqslant 1$. Let $s(v)(\cdot)$ be a minimizer continuous in $v \in[-1,1]$, which must be, obviously, solution of the relaxed problem $\left(\mathscr{R} \mathscr{P}_{v}\right)$ as well. The condition $(2.1)$ gives that $\nabla s(v)(x) \geqslant 0$ a.e. in $\Omega$, if $v \geqslant 0$, and $\nabla s(v)(x) \leqslant 0$ a.e. in $\Omega$ otherwise. More precisely, in the first case $\nabla s(v)(x)$ equals 0 or 1 a.e. in $\Omega$ while in the second one $\nabla s(v)(x)$ admits the values -1 or 0 for a.e. $x \in \Omega$. Then, by the same argument as in the proof of Proposition 2.1 we have

$$
s_{0}^{-}(v)(x) \leqslant s(v)(x) \leqslant s_{0}^{+}(v)(x),
$$

$v \in[-1,1], x \in \bar{\Omega}$, where

$$
\begin{aligned}
& s_{0}^{-}(v)(x):=\max _{x^{\prime}= \pm 1} \inf _{v^{\prime} \in \hat{F}_{0}(v)}\left\{v x^{\prime}+v^{\prime}\left(x-x^{\prime}\right)\right\}, \\
& s_{0}^{+}(v)(x):=\min _{x^{\prime}= \pm 1} \sup _{v^{\prime} \in \hat{F}_{0}(v)}\left\{v x^{\prime}+v^{\prime}\left(x-x^{\prime}\right)\right\}, \\
& \hat{F}_{0}(v):= \begin{cases}{[-1,0]} & \text { if }-1 \leqslant v<0, \\
\{0\} & \text { if } v=0, \\
{[0,1]} & \text { if } 0<v \leqslant 1 .\end{cases}
\end{aligned}
$$

Let us fix, for instance, $0<v \leqslant 1$. Then from (5.12) we obtain, in particular, that

$$
s_{0}^{+}(v)(x)= \begin{cases}-v+x+1 & \text { if }-1 \leqslant x<2 v-1, \\ v & \text { if } 2 v-1 \leqslant x \leqslant 1 .\end{cases}
$$


On the other hand, choosing an integer $k$ with $k v \geqslant 1$ and taking the solution $s_{k}(v)(\cdot)$ of the relaxed problem constructed in Example 2.2, we see that $[-1,-1+1 / k] \subset[-1,2 v-1]$ and

$$
\begin{aligned}
s_{k}(v)(x) & =2 k v(x+1)-v=s_{0}^{+}(v)(x)+(2 k v-1)(x+1) \\
& \geqslant s_{0}^{+}(v)(x)+x+1,
\end{aligned}
$$

whenever $x \in[-1,-1+1 / k]$. Thus, $s_{k}(v)(x)$ cannot be approximated by solutions of the problem $\left(\mathscr{P}_{v}\right)$, uniformly in $x \in \bar{\Omega}$.

This is an extreme case: indeed, as follows from (5.11), the problem $\left(\mathscr{P}_{v}\right)$ admits the unique solution $s(v)(x)=v x$ for $v= \pm 1$, while the relaxed functional has a lot of minimizers.

\section{Well-posedness with the best Lipschitz constant}

Recalling Theorem 2.1 we see that not only the affine function $\langle v, \cdot\rangle$ but also the upper and lower solutions $s^{+}(v)(\cdot), s^{-}(v)(\cdot)$ of the relaxed problem $\left(\mathscr{R} \mathscr{P}_{v}\right)$ satisfy the Lipschitz inequality w.r.t. $v$ with the constant $\|\Omega\|=\max _{x \in \bar{\Omega}}|x|$. Taking into account this fact, one is led to wonder that also the original problem $\left(\mathscr{P}_{v}\right)$ should admit a solution possessing the same property. To prove this, consider the set $\mathscr{S}_{\|\Omega\|}^{* *}$ consisting of all mappings $\rho(\cdot) \in \mathscr{S}^{* *}$ such that $v \mapsto\langle v, \cdot\rangle+\rho(v)(\cdot)$ is Lipschitz continuous with Lipschitz constant $\|\Omega\|$. Let also $\mathscr{C}^{+}$and $\mathscr{C}^{-}$be the positive and negative cones in the space $\mathscr{C}\left(\mathbb{R}^{n}, \mathbf{C}_{0}(\bar{\Omega})\right)$, respectively. Namely,

$$
\begin{aligned}
\mathscr{C}^{+} & :=\left\{\rho(\cdot) \in \mathscr{C}\left(\mathbb{R}^{n}, \mathbf{C}_{0}(\bar{\Omega})\right): \rho(v)(x) \geqslant 0 \forall v \in \mathbb{R}^{n}, x \in \bar{\Omega}\right\}, \\
\mathscr{C}^{-} & :=\left\{\rho(\cdot) \in \mathscr{C}\left(\mathbb{R}^{n}, \mathbf{C}_{0}(\bar{\Omega})\right): \rho(v)(x) \leqslant 0 \forall v \in \mathbb{R}^{n}, x \in \bar{\Omega}\right\} .
\end{aligned}
$$

Obviously, $\mathscr{S}_{\|\Omega\|}^{* *} \cap \mathscr{C}^{+}$and $\mathscr{S}_{\|\Omega\|}^{* *} \cap \mathscr{C}^{-}$are closed nonempty subsets of $\mathscr{S}^{* *}$, and, consequently (see Lemma 3.1), they are complete metrizable spaces w.r.t. the induced topology.

In the remainder of this section we keep all the notations used above. Observe that, given $m=1,2, \ldots$ and $\eta>0$, the set $\mathscr{S}_{\|\Omega\|}^{* *} \cap \mathscr{C}^{+} \cap \mathscr{S}_{m}^{\eta}$ (respectively, $\mathscr{S}_{\|\Omega\|}^{* *} \cap \mathscr{C}^{-} \cap \mathscr{S}_{m}^{\eta}$ ) is open in $\mathscr{S}_{\|\Omega\|}^{* *} \cap \mathscr{C}^{+}$(respectively, $\mathscr{S}_{\|\Omega\|}^{* *} \cap \mathscr{C}^{-}$). Clearly,

$$
\bigcap_{m, k=1}^{\infty}\left(\mathscr{S}_{\|\Omega\|}^{* *} \cap \mathscr{C}^{ \pm} \cap \mathscr{S}_{m}^{1 / k}\right)=\mathscr{S}_{\|\Omega\|}^{* *} \cap \mathscr{C}^{ \pm} \cap \mathscr{S}^{\mathrm{ext}}
$$

Let us prove now the following density statement.

Lemma 6.1. $\mathscr{S}_{\|\Omega\|}^{* *} \cap \mathscr{C}^{ \pm} \cap \mathscr{S}_{m}^{\eta}$ is dense in $\mathscr{S}_{\|\Omega\|}^{* *} \cap \mathscr{C}^{ \pm}$for any $m=1,2, \ldots$ and $\eta>0$.

Proof. Let us omit the index $m$ everywhere in what follows as we did earlier.

Fix $\eta>0, \tilde{\rho}(\cdot) \in \mathscr{S}_{\|\Omega\|}^{* *} \cap \mathscr{C}^{+}$and $\varepsilon>0$. First we set

$$
\bar{\rho}(v)(x):=\min \left\{\tilde{\rho}(v)(x)+\varepsilon / 2, \rho^{+}(v)(x)\right\}, \quad v \in \mathbb{R}^{n}, \quad x \in \bar{\Omega},
$$


and observe that $\bar{\rho}(\cdot) \in \mathscr{S}_{\|\Omega\|}^{* *} \cap \mathscr{C}^{+}$(see Proposition $2.4[12]$ ). Moreover $\bar{\rho}(v)(x)>0$ everywhere in $\Omega$, and

$$
\tilde{\rho}(v)(x) \leqslant \bar{\rho}(v)(x) \leqslant \tilde{\rho}(v)(x)+\varepsilon / 2 \quad \forall v \in \mathbb{R}^{n}, \quad x \in \bar{\Omega} .
$$

By Lemma 5.1 there exists $0<\delta<\varepsilon / 2$ such that

$$
\mu\{x \in \Omega: \bar{\rho}(v)(x)<\delta\}<\eta / D
$$

for all $v \in V^{\eta}$. Repeating the first steps of the proof of Theorem 3.1 (see Section 5) we find points $x_{i}^{+} \in \Omega, i=1, \ldots, N^{+}$, such that (5.4) holds. Let us define the mapping $f: \bar{V} \rightarrow \mathbf{C}(\bar{\Omega})$ by

$$
f(v)(x):=\max _{1 \leqslant i \leqslant N^{+}}\left\{\bar{\rho}(v)\left(x_{i}^{+}\right)-\sigma_{V-v}\left(x_{i}^{+}-x\right)-\delta / 2, \bar{\rho}(v)(x)-\varepsilon / 2,0\right\},
$$

and observe that $v \mapsto\langle v, \cdot\rangle+f(v)(\cdot)$ is Lipschitz continuous with Lipschitz constant $\|\Omega\|$ as a mapping to $\mathbf{C}(\bar{\Omega})$. Furthermore, $f(v)(x)=0$ whenever either $v \in \partial V$ or $x \in \partial \Omega$, and $\nabla f(v)(x) \in \bar{V}-v$ for a.e. $x \in \Omega$. So that the mapping $\hat{\rho}: \mathbb{R}^{n} \rightarrow \mathbf{C}_{0}(\bar{\Omega})$, equal to $f(v)$ for $v \in \bar{V}$ and to $\bar{\rho}(v)$ elsewhere, belongs to $\mathscr{S}_{\|\Omega\|}^{* *} \cap \mathscr{C}^{+}$.

For every $v \in \bar{V}$ and $x \in \bar{\Omega}$ we have $\bar{\rho}(v)\left(x_{i}^{+}\right)-\sigma_{V-v}\left(x_{i}^{+}-x\right) \leqslant \bar{\rho}(v)(x), i=1, \ldots, N^{+}$. Then from (6.4) it obviously follows that $|f(v)(x)-\bar{\rho}(v)(x)| \leqslant \varepsilon / 2$. Combining this with (6.2) we see that $\|\hat{\rho}(v)-\tilde{\rho}(v)\|_{\infty} \leqslant \varepsilon \forall v \in \mathbb{R}^{n}$.

Finally, let us fix $v \in V^{\eta}$ and $x \in \Omega$ with $\bar{\rho}(v)(x) \geqslant \delta$. Then by using (5.4) and (5.3) we choose $i=1, \ldots, N^{+}$such that

$$
\bar{\rho}(v)\left(x_{i}^{+}\right)-\sigma_{V-v}\left(x_{i}^{+}-x\right)>\delta / 2
$$

and

$$
\left|x-x_{i}^{+}\right|<\varepsilon /(8 D) .
$$

We deduce from (6.6) the inequality

$$
\begin{aligned}
\bar{\rho}(v)\left(x_{i}^{+}\right)-\sigma_{V-v}\left(x_{i}^{+}-x\right)-\delta / 2 & \geqslant \bar{\rho}(v)(x)-2 D\left|x-x_{i}^{+}\right|-\delta / 2 \\
& >\bar{\rho}(v)(x)-\varepsilon / 2,
\end{aligned}
$$

which together with (6.5) implies that

$$
f(v)(x)=\max _{1 \leqslant i \leqslant N^{+}}\left\{\bar{\rho}(v)\left(x_{i}^{+}\right)-\sigma_{V-v}\left(x_{i}^{+}-x\right)-\delta / 2\right\} .
$$

Therefore (see Proposition 2.4 [12] and Corollary 25.1.3 [17]) we have $\nabla \hat{\rho}(v)(x)=\nabla f(v)(x)$ $\in \operatorname{ext} V-v$ a.e. in $\Omega$. Hence, by the choice of $\delta$ (see (6.3)), we get $\hat{\rho}(\cdot) \in \mathscr{S}^{\eta}$, and density of the set $\mathscr{S}_{\|\Omega\|}^{* *} \cap \mathscr{C}^{+} \cap \mathscr{S}^{\eta}$ in $\mathscr{S}_{\|\Omega\|}^{* *} \cap \mathscr{C}^{+}$follows.

The case of the set $\mathscr{S}_{\|\Omega\|}^{* *} \cap \mathscr{C}^{-}$may be treated similarly.

Finally, using the last lemma combined with (6.1) and applying the Baire category theorem, we obtain the following result. 
Theorem 6.1. The variational problem $\left(\mathscr{P}_{v}\right)$ admits a minimizer $\hat{s}(v)(\cdot)$, defined in the set of those $v$ where the minimum exists, which is $\|\Omega\|$-Lipschitz continuous (i.e. Lipschitz continuous w.r.t. $v$ with Lipschitz constant $\left.\|\Omega\|=\max _{x \in \bar{\Omega}}|x|\right)$. Moreover, this minimizer can be chosen arbitrarily near to any $\|\Omega\|$-Lipschitz continuous solution $\tilde{s}(v)(\cdot)$ of the relaxed problem $\left(\mathscr{R} \mathscr{P}_{v}\right)$ which always remains above (respectively, below) the affine function.

Remark 6.1. In particular, there exists a Lipschitz continuous selection inside any neighbourhood of the affine function $v \mapsto\langle v, \cdot\rangle$. Notice also that in [10] we have proved a constructive version of the Vitali covering theorem, with which we have constructed a minimizer having Lipschitz constant $\|\Omega\|+3$; while now, in Theorem 6.1, this constant has been improved to $\|\Omega\|$ (which is the best possible Lipschitz constant).

\section{Some remarks on gradient inclusions}

Let us notice once more that we search (in the proofs of Theorems 2.1 and 3.1) solutions of the problem $\left(\mathscr{R} \mathscr{P}_{v}\right)$ or $\left(\mathscr{P}_{v}\right)$ among the functions $u(\cdot)$ satisfying the relation $(2.1)$ (or $\nabla u(x) \in \operatorname{ext} \hat{F}(v)$, respectively) for a.e. $x \in \Omega$. See also the Example 5.1 which shows that generally our technique is not applicable and the results fail if the variational problem cannot be reduced to the inclusions above (for instance, with unbounded $n$-dimensional faces). On the other hand, whenever such reduction is possible we have obtained in fact a result which is stronger than the one given by Theorem 3.1. Indeed, in order to be solution of $\left(\mathscr{P}_{v}\right)$ a function $u(\cdot)$ does not need to have its gradients on the extremal boundary of $\hat{F}(v)$ but should satisfy the weaker condition $g(\nabla u(x))=g^{* *}(\nabla u(x))$. Taking into account these observations we can formulate our existence and density result for solutions of gradient inclusions. Namely, let us consider a convex compact set $F \subset \mathbb{R}^{n}$ and an open bounded domain $\Omega \subset \mathbb{R}^{n}$. From the proof of Theorem 3.1 we obtain immediately the corollary below which describes the structure of the solution set to the following boundary value problems:

$$
\begin{array}{lr}
\nabla u(x) \in F,\left.\quad u\right|_{\partial \Omega}=\langle v, \cdot\rangle ; & \left(\mathscr{R}_{v}^{\prime}\right) \\
\nabla u(x) \in \operatorname{ext} F,\left.\quad u\right|_{\partial \Omega}=\langle v, \cdot\rangle . & \left(\mathscr{P}_{v}^{\prime}\right)
\end{array}
$$

Notice that by solution of $\left(\mathscr{R} \mathscr{P}_{v}^{\prime}\right)$ or $\left(\mathscr{P}_{v}^{\prime}\right)$ we mean any Lipschitz continuous function $u: \bar{\Omega} \rightarrow \mathbb{R}$ (with Lipschitz constant $\|F\|:=\max _{v \in F}|v|$ ) satisfying the condition $u(x)=$ $\langle v, x\rangle \quad \forall x \in \partial \Omega$ and such that the respective gradient inclusion holds for a.e. $x \in \Omega$. Notice the connection between the differential inclusions $\left(\mathscr{R} \mathscr{P}_{v}^{\prime}\right)$ and $\left(\mathscr{P}_{v}^{\prime}\right)$, on one hand, and, on the other hand, the variational problems $\left(\mathscr{R} \mathscr{P}_{v}\right)$ and $\left(\mathscr{P}_{v}\right)$ with lagrangian

$$
g(v)= \begin{cases}0 & \text { if } v \in \overline{\operatorname{ext}} F \\ +\infty & \text { otherwise }\end{cases}
$$

\section{Corollary 7.1.}

(i) For any $v \in F$, each solution $u(\cdot)$ of $\left(\mathscr{R} \mathscr{P}_{v}^{\prime}\right)$ satisfies the inequalities

$$
u_{v}^{-}(x) \leqslant u(x) \leqslant u_{v}^{+}(x) \quad \forall x \in \bar{\Omega},
$$


where

$$
\begin{aligned}
& u_{v}^{+}(x):=\inf _{x^{\prime} \in \partial \Omega} \sup _{v^{\prime} \in F}\left\{\left\langle v, x^{\prime}\right\rangle+\left\langle v^{\prime}, x-x^{\prime}\right\rangle\right\}, \\
& u_{v}^{-}(x):=\sup _{x^{\prime} \in \partial \Omega} \inf _{v^{\prime} \in F}\left\{\left\langle v, x^{\prime}\right\rangle+\left\langle v^{\prime}, x-x^{\prime}\right\rangle\right\} .
\end{aligned}
$$

(ii) The functions $u_{v}^{-}(x)$ and $u_{v}^{+}(x)$ are themselves solutions (minimal and maximal) of $\left(\mathscr{R} \mathscr{P}_{v}^{\prime}\right)$. Furthermore, $v \mapsto u_{v}^{-}(\cdot)$ and $v \mapsto u_{v}^{+}(\cdot)$ are Lipschitz continuous with Lipschitz constant $\|\Omega\|:=\max _{x \in \bar{\Omega}}|x|$, as mappings from $F$ into $\mathbf{C}(\bar{\Omega})$.

(iii) For each solution $\tilde{u}_{v}(\cdot)$ of $\left(\mathscr{R}_{P^{\prime}}^{\prime}\right)$ continuously depending on $v \in$ int $F$ w.r.t. the norm in $\mathbf{C}(\bar{\Omega})$ and each $\varepsilon>0$ there exists a solution $\hat{u}_{v}(\cdot)$ of $\left(\mathscr{P}_{v}^{\prime}\right)$ still continuously depending on $v$, and such that $\left|\hat{u}_{v}(x)-\tilde{u}_{v}(x)\right| \leqslant \varepsilon$ whenever $x \in \bar{\Omega}, v \in \operatorname{int} F$.

Observe also that $u_{v}^{+}(x)-\langle v, x\rangle$ can be interpreted as the minimal time function with the constant dynamics $-(F-v)^{*}$ and the target set $\partial \Omega$ to be reached from inside the domain $\Omega$. Therefore, it follows from Theorem 3.1 [7] that $u_{v}^{+}(\cdot)$ (and, symmetrically, $u_{v}^{-}(\cdot)$ ) cannot have gradients in the interior of $F$. However, for the sake of completeness, let us give here the proof of this simple fact based on the Corollary above.

Proposition 7.1. Let $v \in F$ and $x \in \Omega$ be such that the gradient $\nabla u_{v}^{+}(x)\left(\right.$ or $\left.\nabla u_{v}^{-}(x)\right)$ exists. Then $\nabla u_{v}^{+}(x) \in \partial F$ (respectively, $\nabla u_{v}^{-}(x) \in \partial F$ ).

Proof. Since $u_{v}^{+}(x) \equiv\langle v, x\rangle$ for $v \in \partial F$, we can assume that $v \in$ int $F$. Suppose now that there exists a point $x^{*} \in \Omega$ with $\nabla u_{v}^{+}\left(x^{*}\right) \in$ int $F$, and let $\varepsilon>0$ be such that $\nabla u_{v}^{+}\left(x^{*}\right)+\varepsilon \overline{\mathbf{B}} \subset$ $F$. Then for some $\eta>0$ small enough we have

$$
\left|u_{v}^{+}(x)-u_{v}^{+}\left(x^{*}\right)-\left\langle\nabla u_{v}^{+}\left(x^{*}\right), x-x^{*}\right\rangle\right|<1 / 4 \varepsilon \eta,
$$

whenever $x \in \Omega$ with $\left|x-x^{*}\right| \leqslant \eta$. Let us define the function $h: x^{*}+\eta \overline{\mathbf{B}} \rightarrow \mathbb{R}$ by

$$
h(x):=\max \left\{u_{v}^{+}\left(x^{*}\right)-u_{v}^{+}(x)-\left\langle\nabla u_{v}^{+}\left(x^{*}\right), x^{*}-x\right\rangle+\frac{\varepsilon}{4} \eta \omega\left(\frac{\left|x-x^{*}\right|}{\eta}\right), 0\right\},
$$

where the piecewise affine function $\omega: \mathbb{R}^{+} \rightarrow[-1,1]$,

$$
\omega(t):= \begin{cases}1 & \text { if } 0 \leqslant t \leqslant 1 / 2 \\ 3-4 t & \text { if } 1 / 2<t<1 \\ -1 & \text { if } t \geqslant 1\end{cases}
$$

admits derivative equal to 0 or -4 for all $t \neq 1 / 2,1$. We assume, of course, that $x^{*}+\eta \overline{\mathbf{B}} \subset$ $\Omega$. If $\left|x-x^{*}\right| \leqslant \eta / 2$ then by (7.4) and (7.3)

$$
0<u_{v}^{+}\left(x^{*}\right)-u_{v}^{+}(x)-\left\langle\nabla u_{v}^{+}\left(x^{*}\right), x^{*}-x\right\rangle+\frac{\varepsilon}{4} \eta=h(x) .
$$

Furthermore, on the boundary of $x^{*}+\eta \overline{\mathbf{B}}$ we have $\omega\left(\left|x-x^{*}\right| / \eta\right)=-1$ and (7.3) gives $h(x)=0$. Therefore, $h(\cdot)$ can be extended continuously by setting $h(x)=0$ for all $x \in \bar{\Omega}$ 
with $\left|x-x^{*}\right|>\eta$. Then the function $h: \bar{\Omega} \rightarrow \mathbb{R}$ will be Lipschitz continuous, and by the Proposition 2.4 [12] its gradient is equal either to 0 or to

$$
\nabla u_{v}^{+}\left(x^{*}\right)-\nabla u_{v}^{+}(x)+\frac{\varepsilon}{4} \eta \nabla \omega\left(\frac{\left|x-x^{*}\right|}{\eta}\right)
$$

at almost every point $x \in \Omega$. If we define now $\hat{u}: \bar{\Omega} \rightarrow \mathbb{R}$ as $\hat{u}(x)=u_{v}^{+}(x)+h(x), \quad x \in \bar{\Omega}$, then, obviously, $\hat{u}(x)=\langle v, x\rangle \quad \forall x \in \partial \Omega$, and the gradient $\nabla \hat{u}(x)$ either equals $\nabla u_{v}^{+}(x)$, or satisfies the inequality $\left|\nabla \hat{u}(x)-\nabla u_{v}^{+}(x)\right| \leqslant \varepsilon$ (see (7.4)). In each case $\nabla \hat{u}(x) \in F$, and $\hat{u}(\cdot)$ is a solution of $\left(\mathscr{R}_{v}^{\prime}\right)$. On the other hand, by (7.5) $\hat{u}(x)>u_{v}^{+}(x)$ for all $x \in \Omega,\left|x-x^{*}\right| \leqslant \eta / 2$, thus contradicting Corollary 7.1 (i).

The function $u_{v}^{-}(\cdot)$ can be treated similarly.

The following simple example shows that, in general, upper and lower solutions (7.1), (7.2) are not solutions of the extremal inclusion $\left(\mathscr{P}_{v}^{\prime}\right)$. That is, the gradients $\nabla u_{v}^{+}(x)$ and $\nabla u_{v}^{-}(x)$ can belong to relative interiors of $k$-dimensional faces of $F$ for $1 \leqslant k \leqslant n-1$ (in the case $n>1)$.

Example 7.1. Let $n=2$ and $F=\bar{\Omega}:=\left\{\left(\xi_{1}, \xi_{2}\right): \max \left(\left|\xi_{1}\right|,\left|\xi_{2}\right|\right) \leqslant 1\right\}$.

Since

$$
F^{*}=\left\{\left(x_{1}, x_{2}\right):\left|x_{1}\right|+\left|x_{2}\right| \leqslant 1\right\},
$$

and $\sigma_{F}\left(x_{1}, x_{2}\right)$ is the gauge function associated to $F^{*}$, it follows from (7.1) that

$$
\begin{gathered}
u_{v}^{+}(x)=\min \left\{\min _{x_{1}^{\prime}= \pm 1,-1 \leqslant x_{2}^{\prime} \leqslant 1}\left(v_{1} x_{1}^{\prime}+v_{2} x_{2}^{\prime}+\left|x_{1}-x_{1}^{\prime}\right|+\left|x_{2}-x_{2}^{\prime}\right|\right),\right. \\
\left.\min _{2= \pm 1,-1 \leqslant x_{1}^{\prime} \leqslant 1}\left(v_{1} x_{1}^{\prime}+v_{2} x_{2}^{\prime}+\left|x_{1}-x_{1}^{\prime}\right|+\left|x_{2}-x_{2}^{\prime}\right|\right)\right\} .
\end{gathered}
$$

After calculations we find

$$
u_{v}^{+}(x)=1+\min \left\{v_{1} x_{1}-\left|x_{2}-v_{2}\right|, v_{2} x_{2}-\left|x_{1}-v_{1}\right|\right\},
$$

and the gradient $\nabla u_{v}^{+}(x)$ equals $\left(v_{1}, \pm 1\right)$ or $\left( \pm 1, v_{2}\right)$ a.e. in $\Omega$, for $v=\left(v_{1}, v_{2}\right) \in$ int $F$. Similarly, the formula (7.2) gives

$$
u_{v}^{-}(x)=-1+\max \left\{v_{1} x_{1}+\left|x_{2}+v_{2}\right|, v_{2} x_{2}+\left|x_{1}+v_{1}\right|\right\},
$$

and also $\nabla u_{v}^{-}(x) \in\left\{\left(v_{1}, \pm 1\right),\left( \pm 1, v_{2}\right)\right\}$ for a.e. $x \in \Omega$. Thus the "extremal" solutions $u_{v}^{+}(\cdot), u_{v}^{-}(\cdot)$ do not satisfy the inclusion $\left(\mathscr{P}_{v}^{\prime}\right)$ on a set of full measure (for each $v=\left(v_{1}, v_{2}\right) \in$ int $F$ ).

\section{References}

[1] A. Bressan, The most likely path of a differential inclusion, J. Differential Equations 88 (1990) 155-174.

[2] P. Cardaliaguet, B. Dacorogna, W. Gangbo, N. Georgy, Geometric restrictions for the existence of viscosity solutions, Ann. Inst. Henri Poicaré 16 (1999) 189-220. 
[3] A. Cellina, On minima of a functional of the gradient: necessary conditions, Nonlinear Anal. Theory Meth. Appl. 20 (1993) 337-341.

[4] A. Cellina, On minima of a functional of the gradient: sufficient conditions, Nonlinear Anal. Theory Meth. Appl. 20 (1993) 343-347.

[5] A. Cellina, S. Zagatti, A version of Olech's Lemma in a problem of the calculus of variations, SIAM J. Contr. Optimiz. 32 (1994) 1114-1127.

[6] F.H. Clarke, Optimization and Nonsmooth Analysis, Willey-Interscience, New York, 1983.

[7] G. Colombo, P.R. Wolensky, Variational analysis for a class of minimal time functions in Hilbert spaces, J. Convex Anal. 11 (2004) 335-361.

[8] M.G. Crandall, P.L. Lions, Viscosity solutions of Hamilton-Jacobi equations, Trans. Am. Math. Soc. 277 (1983) $1-42$.

[9] B. Dacorogna, Direct Methods in the Calculus of Variations, Springer, Berlin, 1989.

[10] G. Dal Maso, V. Goncharov, A. Ornelas, A Lipschitz selection from the set of minimizers of a nonconvex functional of the gradient, Nonlinear Anal. Theory Meth. Appl. 37 (1999) 707-717.

[11] F.S. De Blasi, G. Pianigiani, Differential inclusions in Banach spaces, J. Differential Equations 66 (1987) $208-229$.

[12] F.S. De Blasi, G. Pianigiani, On the Dirichlet problem for first order partial differential equations. A Baire category approach, NoDEA 6 (1999) 13-34.

[13] H. Federrer, Geometric Measure Theory, Springer, Berlin, 1969.

[14] G. Friesecke, A necessary and sufficient condition for nonattainment and formation of microstructure almost everywhere in scalar variational problems, Proc. R. Soc. Edinburgh 124A (1994) 437-471.

[15] V. Goncharov, A. Ornelas, On minima of a functional of the gradient: a continuous selection, Nonlinear Anal. Theory Meth. Appl. 27 (1996) 1137-1146.

[16] D. Kinderlehrer, G. Stampacchia, An Introduction to Variational Inequalities and their Applications, Academic Press, New York, 1980.

[17] R.T. Rockafellar, Convex Analysis, Princeton University Press, Princeton, NJ, 1972.

[18] W. Rudin, Principles of Mathematical Analysis, McGraw-Hill, New York, 1976.

[19] H.H. Schaefer, Topological Vector Spaces, Macmillan, New York, 1966.

[20] M. Sychev, Characterization of homogeneous scalar variational problems solvable for all boundary data, Proc. R. Soc. Edinburgh 130A (2000) 611-631.

[21] S. Zagatti, Minimization of functionals of the gradient by Baire's theorem, SIAM J. Contr. Optimiz. 38 (2000) 384-399. 